\title{
Impacts des rejets accidentels sur la qualité environnementale des sédiments de la rade portuaire d'Abidjan (lagune Ebrié ; Côte d'Ivoire)
}

\author{
Aoua S. COULIBALY ${ }^{1 *}$, Touré MAMADOU ${ }^{1}$, Diangoné ERIC ${ }^{1}$, Sylvain MONDE ${ }^{1}$, \\ Aka KOUAME $^{1}$ et Gérard BLANC ${ }^{2}$ \\ ${ }^{1}$ Université F Houphouet-Boigny, Côte d'Ivoire, UFR-STRM 2 B.P. 582 Abidjan 22 Côte d'Ivoire. \\ ${ }^{2}$ Université Bordeaux, France. \\ *Auteur correspondant, E-mail: aouasougo@yahoo.fr
}

\section{RESUME}

La densité du trafic maritime s'est intensifié depuis la création du port d'Abidjan situé dans l'estuaire de la lagune Ebrié en Côte d'Ivoire. En outre sa rade portuaire est le lieu de rejets industriels et urbains. Les sédiments de la rade portuaire d'Abidjan, ont été analysés par spectrométrie d'adsorption en vue de déterminer les teneurs en éléments traces métalliques (ETM), afin d'évaluer le facteur d'enrichissement et la qualité du sédiment. Les sédiments de ce fond lagunaire ne sont pas pollués en $\mathrm{Cd}$, et sont de bonne qualité environnementale. Leur teneur en $\mathrm{Pb}$ ne constitue pas de danger pour le biota. La pollution en $\mathrm{Cr}, \mathrm{Ni}$ et $\mathrm{Cu}$ est modéré et les sédiments sont de mauvaises qualité. Concernant l'arsenic et le zinc, les échantillons dont le facteur d'enrichissement est inférieur à 1,3 ne constituent pas de danger pour l'environnement. Pour le reste des échantillons, l'arsenic (As) fortement enrichis, présente un danger pour les sédiments. Il en est de même pour $\mathrm{Pb}$.

(C) 2014 International Formulae Group. All rights reserved.

Mots clés : ETM; Facteur d'enrichissement, qualité environnementale, rade portuaire, Abidjan, Côte d'Ivoire

\section{INTRODUCTION}

La rade portuaire d'Abidjan se situe dans l'estuaire de la lagune Ebrié en Côte d'Ivoire. Cette lagune s'étend sur $125 \mathrm{~km}$ de littoral ivoirien et occupe une surface d'environ $500 \mathrm{~km}^{2}$, avec $4 \mathrm{~m}$ de profondeur en moyenne. C'est la plus grande lagune d'Afrique de l'Ouest (Affian ; 2003 ; Mondé 2004). Depuis la création du port d'Abidjan, la densité du trafic maritime est de plus en plus accrue dans sa rade. Il s'en est suivi une forte concentration urbaine et industrielle à l'origine de rejets dans cette zone portuaire (Soro et al., 2009). La lagune Ebrié montre des signes de pollution caractérisés par le phénomène d'eutrophisation et périodiquement par des morts massives de ressources halieutiques (Mondé et al., 2011). L'impact de ces pollutions sur la faune lagunaire et le cadre de vie des populations riveraines, soulignent l'urgence de trouver des solutions à cette situation qui amenuiserait les potentialités économiques du port d'Abidjan.

La présente étude analyse les teneurs en éléments traces métalliques (ETM) des sédiments en déterminant le facteur d'enrichissement. Cette approche permettrait 
d'évaluer la qualité environnementale de la rade portuaire d'Abidjan.

\section{MATERIEL ET METHODES}

\section{Echantillonnage}

Les échantillons de sédiment de surface ont été prélevés dans la rade portuaire (Figure 1) à l'aide d'une benne à sédiment de type Van Veen. Les 6 points échantillonnés ont été repérés par un GPS et couvre l'ensemble de la rade portuaire. Une partie du sédiment est prélevé au cœur de la benne, pour éviter toute contamination métallique. Les échantillons ont été conservés dans des flacons de $50 \mathrm{ml}$. Avant toute utilisation, ce flaconnage a été décontaminé selon le protocole de Coulibaly et al. (2010).

\section{Protocole d'extraction}

$\mathrm{Au}$ laboratoire, les échantillons ont été mis à l'étuve $\left(50{ }^{\circ} \mathrm{C}\right)$ jusqu'à séchage complet. Ils ont été ensuite broyés et homogénéisés dans un mortier en agate manuel. Pour chaque extraction, des blancs de réactifs ont été réalisés; ces blancs ont ensuite été soustraits des concentrations de métaux. Le dosage des métaux s'est effectué sur la fraction inférieure à $63 \mu \mathrm{m}$ suivant la méthode utilisée par Audry et al. (2004).

Une quantité de sédiments $(30 \mathrm{mg})$ est digérée à l'eau régale $(750 \mu \mathrm{HCl}$ « suprapur » à $30,9 \%+250 \mu 1 \mathrm{HNO}_{3}$ « suprapur » à $65,5 \%$ ) et $2 \mathrm{ml}$ de $\mathrm{HF}$ « suprapur » à $65 \%$ dans des flacons en téflon fermés hermétiquement et maintenus pendant $2 \mathrm{~h}$ à $110{ }^{\circ} \mathrm{C}$ sur une plaque chauffante. Après refroidissement, les gouttelettes condensées sur les bouchons des flacons ont été récupérées à l'aide d'un rinçage à l'eau MilliQ. L'évaporation du contenu des flacons a été réalisée par chauffage à $100{ }^{\circ} \mathrm{C}$ pendant $12 \mathrm{~h}$. On a laissé refroidir de nouveau pour effectuer la reprise du résidu de digestion avec $250 \mu \mathrm{l}$ de $\mathrm{HNO}_{3}$ à $65,5 \%$ et $5 \mathrm{ml}$ d'eau Milli-Q. La nouvelle solution est chauffée une dernière fois à $80 \quad{ }^{\circ} \mathrm{C}$ pendant $10 \mathrm{~min}$. Après refroidissement et décantation, $3,5 \mathrm{ml}$ de surnageant ont été prélevés et conservés dans un flacon en polypropylène auquel on a ajouté 6,5 ml d'eau Milli-Q.

\section{Dosage des éléments traces métalliques par ICP-MS (Inductively Coupled Plasma - Mass Spectroscopy)}

L'analyse des ETM ( $\mathrm{Cr}, \mathrm{Ni}, \mathrm{Cu}, \mathrm{Zn}$, $\mathrm{As}, \mathrm{Cd}, \mathrm{Pb})$ a été réalisée à l'aide d'un spectromètre de masse couplé à un plasma d'ionisation (ICP-MS pour Inductively Coupled Plasma - Mass Spectrometer (Perkin-Elmer type Elan 5000)), permettant de détecter des éléments en trace à des concentrations minimales de $10^{-3} \mu \mathrm{g} .1^{-1}$. Les analyses ont été réalisées en mode quantitatif.

\section{RESULTATS}

Teneurs des sédiments en éléments traces métalliques

Le Tableau 1, présente les concentrations en ETM des sédiments de la rade portuaire d'Abidjan. Dans ce tableau, à titre de comparaison, les valeurs métalliques crustales (UCC) (Soto-Jiménez and PaezOsuma, 2001 ; Pekey, 2006) ainsi que celle de l'échantillon A (Coulibaly et al., 2008, 2009) sont présentés. Le choix de cet échantillon A, a été fait, après l'étude d'une trentaine d'échantillon répartis sur la lagune Ebrié (Coulibaly et al., 2009). Il peut être considéré comme un échantillon de référence non pollué, avec des teneurs en ETM très faible. Ces résultats traduisent que les sédiments renferment des teneurs métalliques significatives. Ils sont donc pollués par rapport à nos deux références, mondiale (UCC) et local (échantillon A).

Une analyse statistique (Tableau 1) montre que les concentrations moyennes et même minimales obtenues pour les sédiments de la rade portuaire, sont toutes supérieures à la fois à UCC et aux teneurs obtenues pour l'échantillon A. Pour le cadmium (Cd), les teneurs varient très peu (écart-type ou $\sigma=0,2$ ). Par contre la disparité est beaucoup plus grande pour le zinc $(\sigma=121) . \mathrm{Zn}_{\max }$ est de 395 $\mathrm{mg} / \mathrm{kg}$ et $\mathrm{Zn}_{\min }$ est $89,99 \quad \mathrm{mg} / \mathrm{kg}$. 


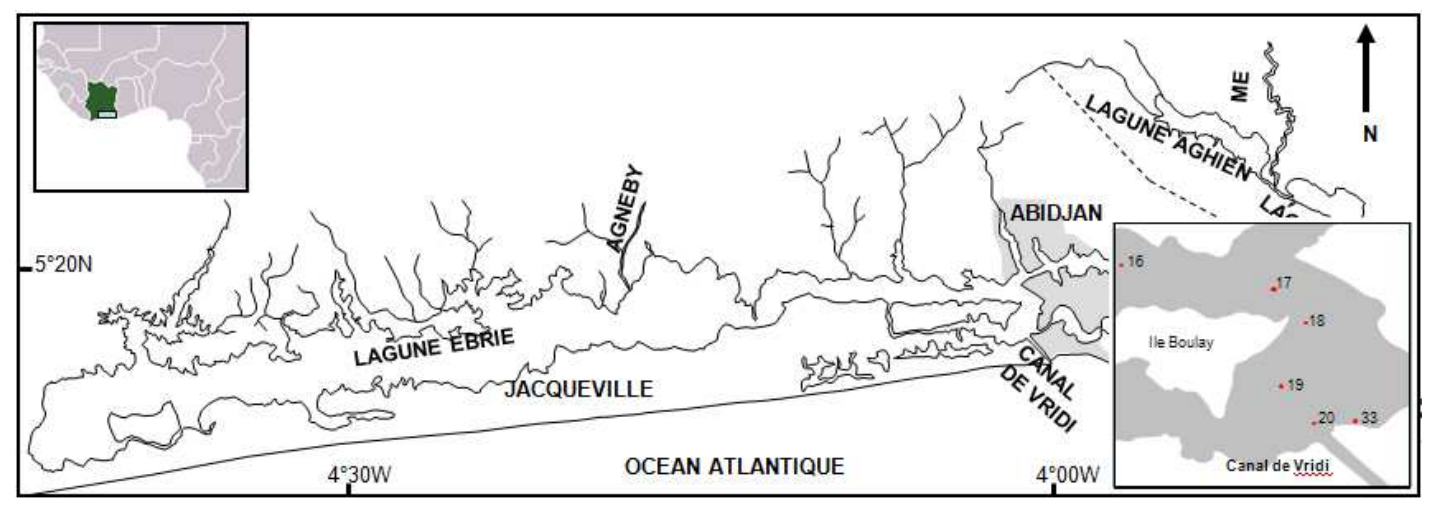

Figure 1: Stations de prélèvement des sédiments dans la rade portuaire d'Abidjan en lagune Ebrié.

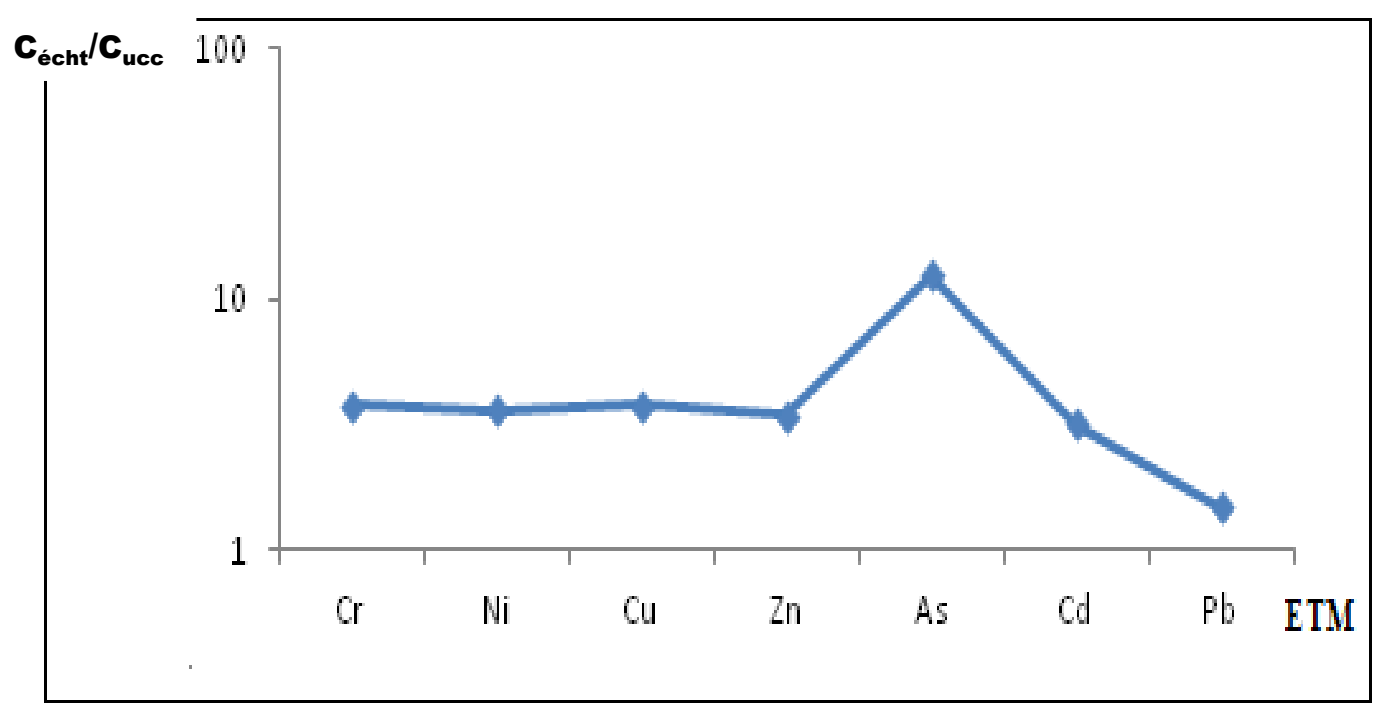

Figure 2 : Proportions relatives des ETM. 

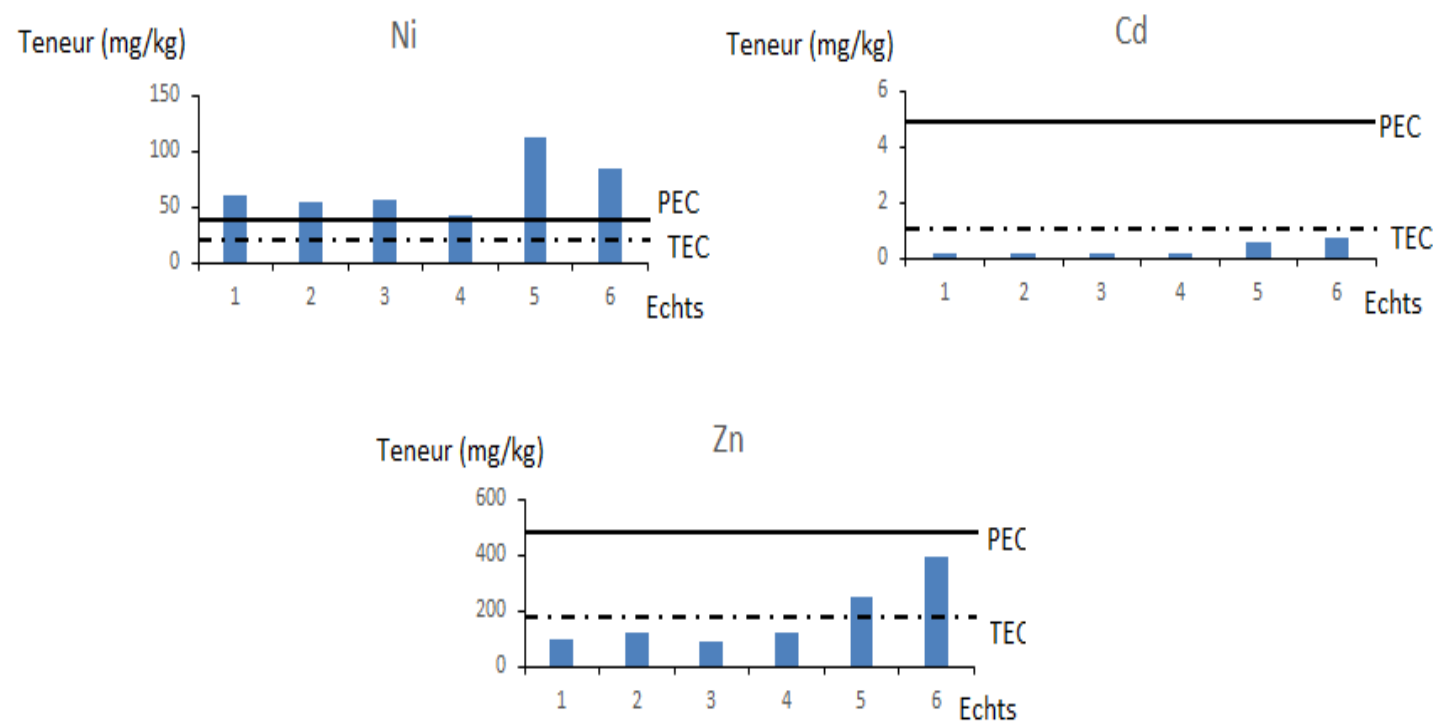

Figure 3 : Evolution des teneurs en $\mathrm{Ni}$; $\mathrm{Cd}$ et $\mathrm{Zn}$ de la rade portuaire par rapport aux TEC et PEC.

Tableau 1 : Teneurs métalliques $(\mathrm{mg} / \mathrm{kg})$ des sédiments de la rade portuaire.

\begin{tabular}{lccccccc}
\hline Stat No & $\mathbf{C r}$ & $\mathbf{N i}$ & $\mathbf{C u}$ & $\mathbf{Z n}$ & $\mathbf{A s}$ & $\mathbf{C d}$ & $\mathbf{P b}$ \\
\hline 1 & 114 & 60,7 & 41,5 & 98 & 8,83 & 0,13 & 18,0 \\
2 & 122 & 53,7 & 35,5 & 123 & 12,6 & 0,18 & 29,6 \\
3 & 144 & 57,0 & 39,8 & 90 & 4,40 & 0,17 & 21,1 \\
4 & 105 & 43,2 & 31,7 & 120 & 26,6 & 0,17 & 28,9 \\
5 & 159 & 113 & 61,4 & 253 & 53,8 & 0,58 & 34,8 \\
6 & 146 & 85,0 & 110 & 395 & 43,4 & 0,69 & 19,63 \\
Min & 105,29 & 43,17 & 31,70 & 89,99 & 4,40 & 0,13 & 18,05 \\
Max & 159 & 113,5 & 110,1 & 395 & 53,78 & 0,69 & 35 \\
Mean & 131,6 & 68,8 & 53,3 & 180 & 24,93 & 0,32 & 25,3 \\
Ecartype & 21 & 25,9 & 29,7 & 121 & 20,0 & 0,2 & 6,7 \\
Ech A & $\mathbf{7 5 , 3}$ & $\mathbf{3 9 , 8}$ & $\mathbf{4 2 , 4}$ & $\mathbf{1 3 0}$ & $\mathbf{6 , 7 9}$ & $\mathbf{0 , 5 8}$ & $\mathbf{2 1 , 0}$ \\
UCC & $\mathbf{3 5}$ & $\mathbf{1 9}$ & $\mathbf{1 4}$ & $\mathbf{5 2}$ & $\mathbf{2}$ & $\mathbf{0 , 1}$ & $\mathbf{1 7}$ \\
\hline
\end{tabular}

Tableau 2 : Facteur d'enrichissement des sédiments de la rade portuaire.

\begin{tabular}{cccccccc}
\hline Stat No & Cr & Ni & \multicolumn{1}{c}{ Cu } & Zn & As & Cd & Pb \\
\hline $\mathbf{1}$ & 1,6 & 1,6 & 1,0 & 0,8 & 1,3 & 0,2 & 0,9 \\
$\mathbf{2}$ & 1,6 & 1,4 & 0,8 & 1,0 & 1,9 & 0,3 & 1,4 \\
$\mathbf{3}$ & 2,5 & 1,9 & 1,2 & 0,9 & 0,8 & 0,4 & 1,3 \\
$\mathbf{4}$ & 2,0 & 1,6 & 1,1 & 1,3 & 5,6 & 0,4 & 2,0 \\
$\mathbf{5}$ & 1,6 & 2,2 & 1,1 & 1,5 & 6,2 & 0,8 & 1,3 \\
$\mathbf{6}$ & 1,3 & 1,5 & 1,8 & 2,1 & 4,4 & 0,8 & 0,6 \\
\hline
\end{tabular}


Tableau 3 : Comparaison des teneurs métalliques par rapport aux TEC et PEC.

\begin{tabular}{lccccccc}
\hline & $\mathbf{C r}$ & $\mathbf{N i}$ & $\mathbf{C u}$ & $\mathbf{Z n}$ & $\mathbf{A s}$ & $\mathbf{C d}$ & $\mathbf{P b}$ \\
\hline TEC & $\mathbf{4 3 , 3}$ & $\mathbf{2 2 , 7}$ & $\mathbf{3 1 , 6}$ & $\mathbf{1 2 1}$ & $\mathbf{9 , 7 9}$ & $\mathbf{0 , 9 9}$ & $\mathbf{3 5 , 6}$ \\
PEC & $\mathbf{1 1 1}$ & $\mathbf{4 8 , 6}$ & $\mathbf{1 4 9}$ & $\mathbf{4 5 9}$ & $\mathbf{3 3}$ & $\mathbf{4 , 9 8}$ & $\mathbf{1 2 8}$ \\
$\leq$ TEC & $0 \%$ & $0 \%$ & $0 \%$ & $50 \%$ & $33 \%$ & $100 \%$ & $100 \%$ \\
Echts & & & & $1 ; 3 ; 4$ & $1 ; 3$ & 1 à 6 & 1 à 6 \\
TEC-PEC & $17 \%$ & $17 \%$ & $100 \%$ & $50 \%$ & $33 \%$ & $0 \%$ & $0 \%$ \\
Echts & 4 & 4 & 1 à 6 & $2 ; 5 ; 6$ & $2 ; 4$ & & \\
$>$ PEC & $83 \%$ & $83 \%$ & $0 \%$ & $0 \%$ & $33 \%$ & $0 \%$ & $0 \%$ \\
Echts & $1 ; 2 ; 3 ; 5 ; 6$ & $1 ; 2 ; 3 ; 5 ; 6$ & & & $5 ; 6$ & & \\
\hline
\end{tabular}

\section{DISCUSSION \\ Normalisation à la croute continentale superficielle}

Dans le but d'examiner la composition globale des sédiments de la rade portuaire, les abondances en ETM de ces sédiments $\left(\mathrm{C}_{\text {écht }}\right)$ ont été normalisées aux concentrations de la croûte continentale superficielle $\left(\mathrm{C}_{\text {ucc }}\right)$ (Figure 2).

La normalisation à la croûte continentale superficielle montre des anomalies positives en la totalité des ETM analysés pour tous les sites de prélèvement. Ces anomalies sont particulièrement prononcées, avec une valeur maximale pour As $(12,47)$ et minimale pour $\mathrm{Pb}(1,49)$. Cela confirme qu'il y a un enrichissement relatif par rapport à la croûte continentale.

\section{Facteur d'enrichissement}

L'évaluation de l'importance d'une pollution métallique nécessite l'utilisation d'un « indice » dont le calcul fait intervenir des concentrations normalisées afin de déconvoluer le signal anthropique du signal «naturel». Pour ce faire, nous avons calculé un facteur d'enrichissement (Enrichment Factor $\mathrm{EF})$ en utilisant une approche géochimique. Elle consiste à séparer la fraction fine $(<63$ $\mu \mathrm{m}$ ), suivie d'une correction géochimique en divisant les teneurs de l'élément étudié par celle d'un élément de référence dont les concentrations sont considérées comme peu ou pas affectées par les phénomènes anthropiques (Roussiez et al., 2005). Les éléments de référence les plus utilisés sont $\mathrm{Al}$, Li, Sc, Cs, Fe (Roussiez et al., 2005 ; Hissler et Probst, 2006 ; Sterckman et al., 2007).

Dans cette étude, les différents facteurs d'enrichissement des ETM dans les sédiments, ont été calculées en utilisant $\mathrm{Al}$ comme élément de référence. Nous avons choisi le sédiment de référence de la lagune Ebrié A (Coulibaly et al., 2008, 2009) comme référence, dans le but d'estimer la pollution locale seule, et non pas inclure dans le facteur d'enrichissement la contribution éventuelle d'autres sources anthropiques, mais cette fois diffuses. Nous avons calculé un facteur d'enrichissement (Enrichment Factor - EF) de la manière suivante :

$\mathrm{EF}=(\mathrm{Me} / \mathrm{Al})$ sed $/(\mathrm{Me} / \mathrm{Al})_{\mathrm{A}}$, avec $M e$ la concentration du métal considéré.

Il est communément admis que $\mathrm{EF}<2$ reflète la variabilité naturelle inhérente à la composition minéralogique de l'échantillon; au-delà, un enrichissement significatif est suspecté (Sutherland, 2000; Hernandez, 2003). Cependant, l'utilisation $\mathrm{du}$ fond géochimique local dans le calcul du facteur d'enrichissement amène à baisser ce seuil à 1,5 , car cette approche atténue fortement le risque de variabilité naturelle du sédiment que pourrait causer les valeurs crustales

Le Tableau 2 présente les valeurs de EF dans les sédiments de la rade portuaire d'Abidjan. Les sédiments de la rade portuaire ne sont pas enrichis en $\mathrm{Cd}(0,2<\mathrm{EF}>0,8)$. Par contre, en ce qui concerne les autres métaux 
étudiés, l'enrichissement est variable tant d'un point de vue qualitatif que quantitatif.

As est l'ETM le plus enrichit dans les sédiments de la rade portuaire. Selon la classification de Sutherland (2000), les échantillons 4,5 et 6 présentent un enrichissement modéré à significatif en As. Le facteur d'enrichissement pour ces échantillons s'élève respectivement à 5,$6 ; 6,2$ et 4,4 . Pour $\mathrm{Cr}$ et $\mathrm{Ni}$, l'enrichissement dans la rade portuaire est faible, et la valeur de EF ne dépasse pas 2,5. Un faible enrichissement $(1,8<\mathrm{EF}>2,1)$ en $\mathrm{Cu}$ et $\mathrm{Zn}$ est noté dans l'échantillon 6 et en $\mathrm{Pb}$ dans l'échantillon 4.

\section{Qualité environnementale des sédiments de la rade portuaire \\ Pour évaluer la qualité} environnementale des sédiments, nous avons comparés les concentrations des ETM de la zone d'étude aux valeurs guides américaines SQGs (Sediment Quality Guidelines) récemment développés à partir d'une base de données d'effets biologiques et écologiques que pourraient occasionnés certaines concentrations de polluants dans les sédiments. Il s'agit du TEC (Threshold Effect Concentration) et du PEC (Probable Effect Concentration) établis par MacDonald et al. (2000) pour évaluer la qualité des sédiments marins et l'eau douce.

Le TEC identifie les concentrations des contaminants en dessous desquels les organismes vivants dans les sédiments ne sont pas affectés, contrairement au PEC, qui lui, identifie les concentrations des contaminants au-dessus desquels on observe des effets néfastes sur les organismes vivants dans les sédiments. Les concentrations comprises entre le TEC et PEC peuvent occasionnellement avoir des effets néfastes sur les organismes. Ces différents travaux ont permis de déterminer les valeurs du TEC et du PEC de 8 métaux traces $(\mathrm{As}, \mathrm{Cd}, \mathrm{Cr}, \mathrm{Cu}, \mathrm{Pb}, \mathrm{Hg}, \mathrm{Ni}$ et $\mathrm{Zn})$.

Les valeurs de ces seuils définis ont été comparées aux teneurs obtenues dans cette étude (Tableau 3). Les sédiments de la rade portuaire d'Abidjan sont de mauvaises qualité en ce qui concernent le $\mathrm{Cr}, \mathrm{Ni}$ et $\mathrm{Cu}$. Ils sont de bonne qualité pour le $\mathrm{Cd}$ et le $\mathrm{Pb}$. Toutefois pour le $\mathrm{Zn}$ et As ; certains sédiments sont de bonne qualité. Il s'agit des échantillons 1 ; 3 et 4 pour le $\mathrm{Zn}$ et ; les échantillons 1 et 3 pour As (Figure 3).

\section{Conclusion}

Les sédiments de la rade portuaire d'Abidjan ont été analysés en vue de déterminer les teneurs en ETM. Les résultats ont permis d'évaluer l'ampleur de la pollution métallique et d'estimer la qualité de ces sédiments.

Les sédiments de la rade portuaire ne sont pas pollués en $\mathrm{Cd}$, et sont de bonne qualité environnementale. Leur teneur en $\mathrm{Pb}$ ne constitue pas de danger pour le biota. La pollution en $\mathrm{Cr}$, $\mathrm{Ni}$ et $\mathrm{Cu}$ est modérés et les sédiments sont de mauvaises qualité. Concernant l'arsenic et le zinc, les échantillons dont le facteur d'enrichissement est inférieur à 1,3 (échantillons 1 et 3 pour As ; 1 ; 3 et 4 pour Zn) ne constituent pas de danger pour l'environnement. Pour le reste des échantillons $(2 ; 4 ; 5 ; 6)$, As fortement enrichis, présente un danger pour les sédiments. Il en est de même pour $\mathrm{Pb}$ (échantillons $2 ; 5$ et 6 ).

\section{REFERENCES}

Affian K. 2003. Approche environnementale d'un écosystème lagunaire microtidal (Lagune Ebrié en Côte d'Ivoire), par des études géochimiques et hydrologiques, bathymétriques et hydrologiques : Contribution du S.I.G. et de la télédétection. Thèse Doctorat, Université Cocody, 225p.

Audry S, Schäfer J, Blanc G, Bossy C, Lavaux G. 2004. Anthropogenic components on heavy metal $(\mathrm{Cd}, \mathrm{Zn}, \mathrm{Cu}$, and $\mathrm{Pb}$ ) budgets in the Lot-Garonne fluvial system (France). Appl. Geochem., 19: 769-86.

Coulibaly AS, Monde S, Wognin VA, Aka K. 2008. State of anthropic pollution in the 
estuary of Ebrié lagoon (Côte d'Ivoire) by analysis of the Metal Elements Traces. European Journal of Scientific Research, 19(2): 372-390.

Coulibaly AS, Monde S, Wognin VA, Aka K. 2009. Analyse des éléments traces métalliques (ETM) dans les baies estuariennes d'Abidjan (Côte d'Ivoire). Afrique Science, 5(3): 77-96.

Coulibaly AS, Monde S, Aka K. 2010. Biodisponibilité et spéciation chimique des éléments traces métalliques dans un environnement confiné : la baie de Biétry. International Journal of African Studies, 3: 41-52.

Hernandez L, Probst A, Probst JL, Ulrich E. 2003. Heavy metal distribution in some French forest soils: evidence for atmospheric contamination. Science of Total Environment, 12:195-219.

Hissler C, Probst JL. 2005 Impact of mercury atmospheric deposition on soils and streams in a mountainous catchment (Vosges, France) polluted by chlor-alkali industrial activity: the important trapping role of the organic matter. Sci. Total Environ., 361:1-3.

Macdonald DD, Ingersoll CG, Berger TA. 2000. Development and evaluation of consensus-based sediment quality guidelines for freshwater ecosystems. Arch. Environ. Con. Tox., 39 : 20-31.

Mondé S. 2004. Etude et modélisation hydrodynamique de la circulation des masses d'eau dans la lagune Ébrié (Côte d'Ivoire). Thèse Doctorat d'État, Université Cocody, 325p.
Monde S, Coulibaly A, Wango T, Aka K. 2011. Simulations prédictives de la qualité des eaux lors des rejets anthropiques dans la lagune Ebrié (Côte d'Ivoire). International Journal of African Studies, 4: 28-40.

Pekey H. 2006. The distribution and sources of heavy metals in Izmit Bay surface sediments affected by polluted straem. Marine Pollution Bulletin, 52: 1197-208.

Roussiez V, Ludwig W, Probst JL, Monaco A. 2005. Background levels of heavy metals in surficial sediments of the Gulf of Lions (NW Mediterranean): an approach based on 133Cs normalization and lead isotope measurements. Environmental Pollution, 138:167-77.

Soro G, Metongo SB, Soro N, Ahoussi KE, Kouamé KF, Zadé SGP, Soro T. 2009. Métaux lourds $(\mathrm{Cu}, \mathrm{Cr}, \mathrm{Mn}$, et $\mathrm{Zn})$ dans les sédiments de surface d'une lagune tropicale africaine. Cas de la lagune Ebrié (Côte d'Ivoire) Int. J. Biol. Chem. Sci., 3(6): 1408-1427.

Summer JK, Wade LD, Engle VD, Malaeb ZA. 1996. Normalization of metal concentrations in estuarie sediments from Gulf of Mexico. Estuaries, 19:581-94.

Sutherland RA. 2000. Bed sedimentassociated trace metals in an urban stream, Oalu, Hawaï. Environmental Geology, 39: 611-627.

Sterkman T, Baize F, Fourrier H, Proix N, Schvartz C. 2007. Référentiel pédogéochimique du nord pas de calais : méthode et principaux résultats. Etude et Gestion des Sols, 14(2): 153-168. 